\title{
Preoperative Cyanoacrylate Embolization of Venous Malformations
}

\author{
Eric J. Monroe ${ }^{1}$ Rush Chewning ${ }^{2}$ Kevin S. H. Koo ${ }^{1}$ Giri Shivaram ${ }^{1}$
}

${ }^{1}$ Section of Interventional Radiology, Department of Radiology, Seattle Children's Hospital and University of Washington, Seattle, Washington, United States

2Division of Vascular and Interventional Radiology, Boston Children's Hospital, Boston, Massachusetts, United States

\author{
Address for correspondence Eric J. Monroe, MD, Department of \\ Radiology, Seattle Children's Hospital, 4800 Sand Point Way NE, M/S \\ R-5417, Seattle, WA 98105, United States \\ (e-mail: eric.monroe@seattlechildrens.org).
}

J Clin Interv Radiol ISVIR 2018;2:42-50

\begin{abstract}
Keywords

- venous malformation

- embolization

- n-butyl cyanoacrylate

Percutaneous sclerotherapy and standalone surgical excision have historically been the mainstay of treatment for pediatric venous malformations (VMs). However, both approaches have significant limitations. Sclerotherapy often requires multiple treatment sessions, with each round adding additional procedural and anesthetic risks as well as radiation exposure. Standalone surgical excision of VMs can be complicated by high-volume blood loss, and indistinct lesion margins can lead to incomplete resection, resulting in recurrence. An alternative to these approaches is percutaneous cyanoacrylate glue embolization followed by immediate surgical excision, a single-stage procedure that allows for better surgical margin definition and reduced intraoperative blood loss, potentially leading to more definitive treatment. The authors describe the interventional radiology aspects of this combined procedure, emphasizing materials preparation and embolization technique.
\end{abstract}

\section{Introduction}

Venous malformations (VMs), with a prevalence of 1 to $4 \%$, represent the most common type of congenital vascular anomaly. ${ }^{1-3}$ These are slow-flow lesions consisting of dysplastic venous channels or sacs that lack normal connections to the systemic venous network. VMs are typically soft and compressible, often becoming engorged during exercise, when in a dependent position, or during Valsalva's maneuver. Sluggish or stagnant flow promotes intralesional thrombus. Pain is overwhelmingly the most common presenting symptom, though patients may also have swelling, fullness, bleeding, and limited range of motion when the lesion extends across a joint.

While conservative measures such as compression garments or nonsteroidal anti-inflammatory agents such as aspirin (which reduces thrombus formation) can be helpful, invasive therapy is required for patients with severe symptoms. For decades, surgical excision was the primary treatment, despite the challenges of poorly defined lesion margins and often high-volume intraoperative blood loss. Sclerotherapy has emerged as a minimally invasive alternative, ${ }^{1,4-8}$ but repeated treatments are frequently necessary due to recurrent or persistent symptoms, increasing costs, anesthetic risks, and radiation exposure.

A new single-stage, combined surgical and interventional radiology treatment approach for head and neck VMs was described in 2013 by investigators at Seattle Children's Hospital. ${ }^{9}$ Patients underwent single-stage preoperative $n$-butyl cyanoacrylate ( $n$-BCA) glue embolization of their VMs followed immediately by surgical excision. Patients enjoyed excellent symptom relief, and the challenges of surgical excision were reduced. Early experience redemonstrated efficacy when applied to truncal and extremity VMs. ${ }^{10}$ While embolization of cavitary VMs resulted in expanded, solid masses and gratifying procedures for both the interventionalist and surgeon, the technique performed well across a variety of venous malformations including infiltrative and dysplastic morphologies.

\section{Technique}

\section{Cyanoacrylates}

The basic monomer structure of a cyanoacrylate features two carbon groups with a relatively positive methylene group $\left(=\mathrm{CH}_{2}\right)$ bound to a carbonyl group. Interaction of an anion, such received

October 16, 2017

accepted after revision

December 15, 2017
DOI https://doi.org/

$10.1055 / \mathrm{s}-0038-1642106$.

ISSN 2457-0214.
Copyright $\odot 2018$ by Indian Society of Vascular and Interventional Radiology
License terms

()(1) $\Theta \circledast$ 
as the various compounds found in blood, with the positive methylene group forms a stable but reactive carbanion. The induced negativity in the carbonyl group attacks and strongly binds to the methylene group of the next cyanoacrylate monomer, and the polymerization cascade continues. Long, strong polymer chains are formed. Various hydrocarbons attached to the carbonyl group determine the name and properties of the cyanoacrylate, ranging from commercially available ethyl-2-cyanoacrylate (Krazy Glue, Elmer's Products) to the medical adhesives $n$-BCA and 2-hexyl cyanoacrylate.

Histoacryl (B. Braun) and Trufill (Codman) are both $n$ BCAs widely available in the Unites States with approval by the Food and Drug Administration (FDA) for wound closure and arteriovenous malformation embolization, respectively. Unadulterated $n$-BCA is a low-viscosity, clear, radiolucent liquid. Although a variety of powdered metals and iodized solutions may be added to $n$-BCA for radiopacity and manipulation of the polymerization rate, the authors exclusively use ethiodized oil for these purposes. Lipiodol (Guerbet USA) provides radiopacity ( - Fig. $\mathbf{1}$ ) and a near linear relationship of dilution to polymerization rate for commonly used formulations. ${ }^{11,12}$ Though glue-to-oil ratios varying from $1: 1$ to $1: 4$ are commonly used for arteriovenous malformations, the slower flow of VMs affords greater dilution for longer polymerization times and more thorough lesion filling. The authors plan a default mixture of 1:4 for typical untreated VMs (-Fig. 2). More dilute formulations (1:5-1:6) promote penetration into fine channels of lesions previously treated with sclerotherapy ( - Fig. 3). Rarely, a more concentrated mixture is made if the accessed channel of the malformation exhibits a short or broad connection to a normal conducting vein ( - Fig. 4).

\section{Equipment Preparation}

Cyanoacrylate polymerization begins whether contact with an ionic solution was intentional or unintentional. Preservation of the "ion-free" environment should receive the same meticulous attention as preservation of the sterile field. A separate small table serves this purpose. Interventionalists or technologists handling items from this area must replace their gloves if they have previously touched items from the main table or procedural field. With sufficient personnel available, a dedicated "ion-free" assistant adds efficiency while minimizing costly and frustrating embolic contamination.

The authors' standard glue table organization is depicted (-Fig. 5). Nonheparinized 5\% dextrose in water (D5W) serves

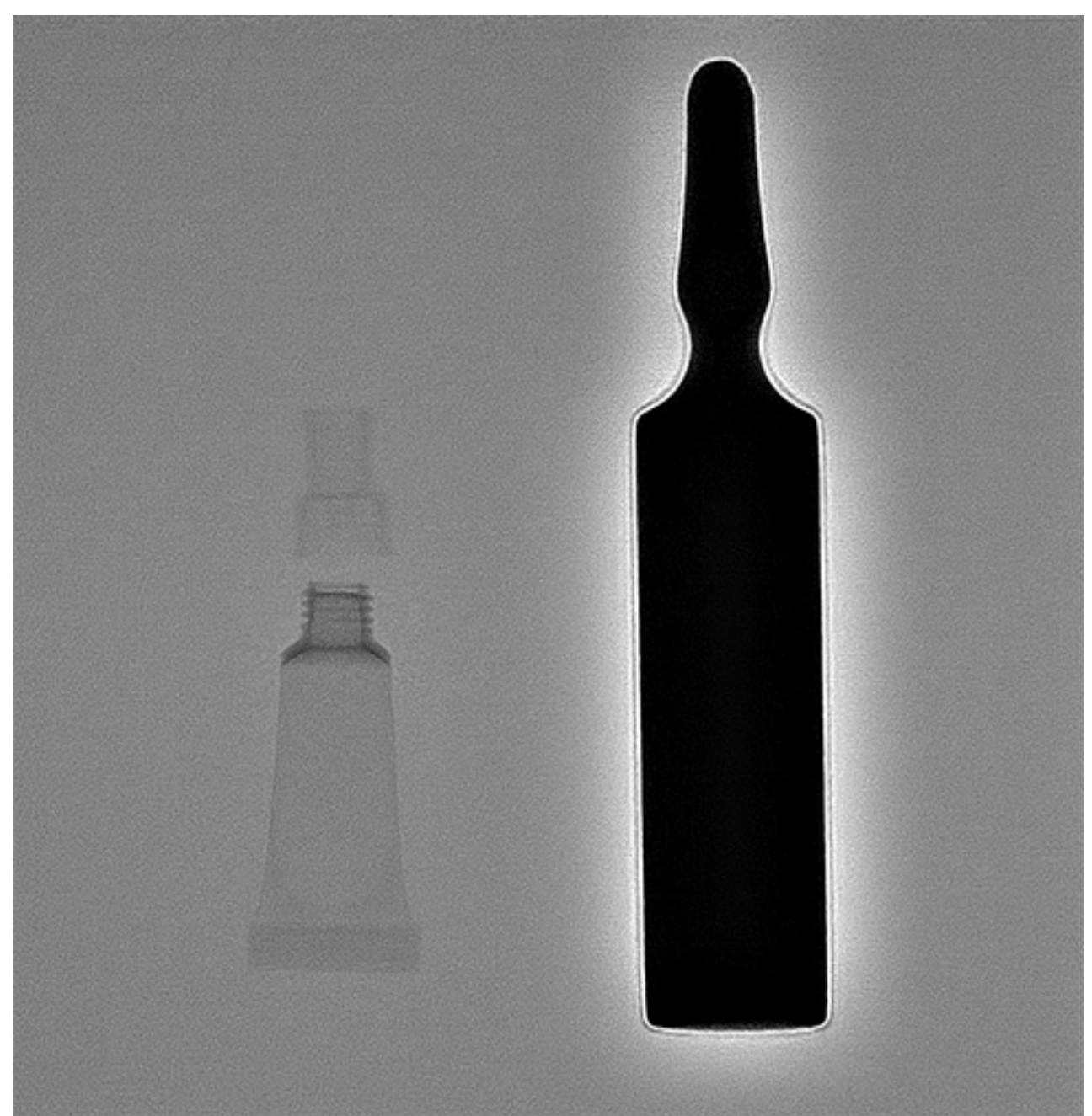

Fig. 1 Fluoroscopic image of unopened radiolucent n-butyl cyanoacrylate (n-BCA) glue (left) (Trufill; Codman) and radiopaque ethiodized oil (right) (Lipiodol; Guerbet USA). 

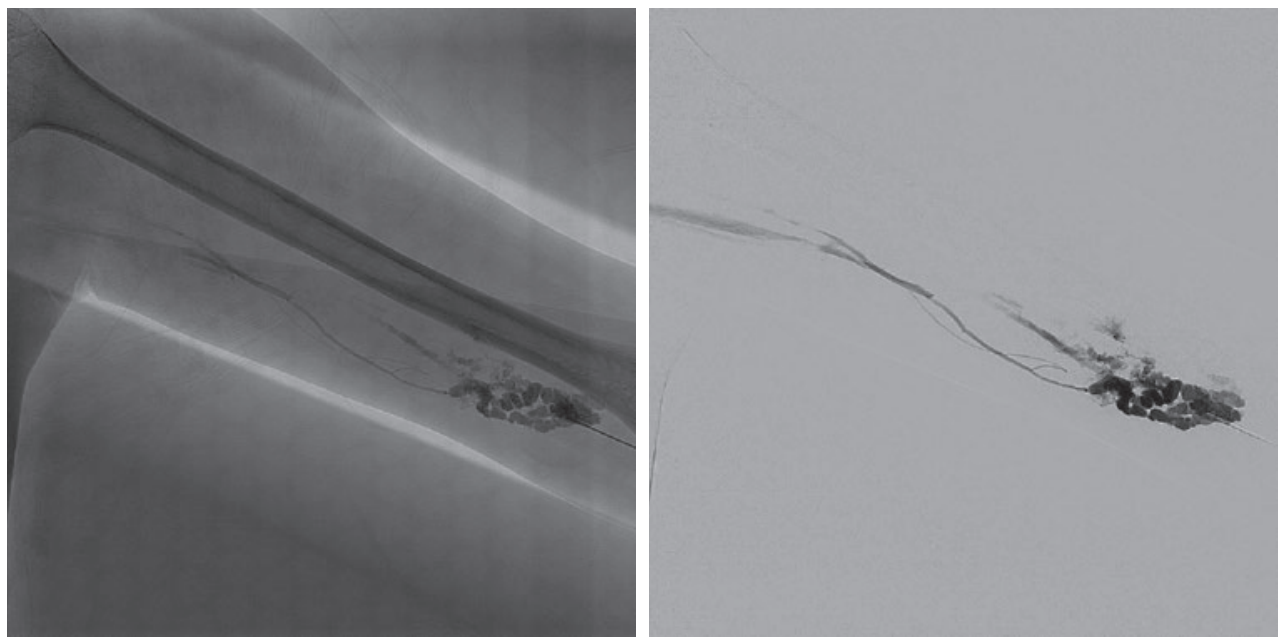

Fig. 2 A 17-year old boy with a painful upper extremity venous malformation. Unsubtracted (a) and subtracted angiographic (b) images demonstrate a typical lesional appearance with drainage to basilic and brachial veins.

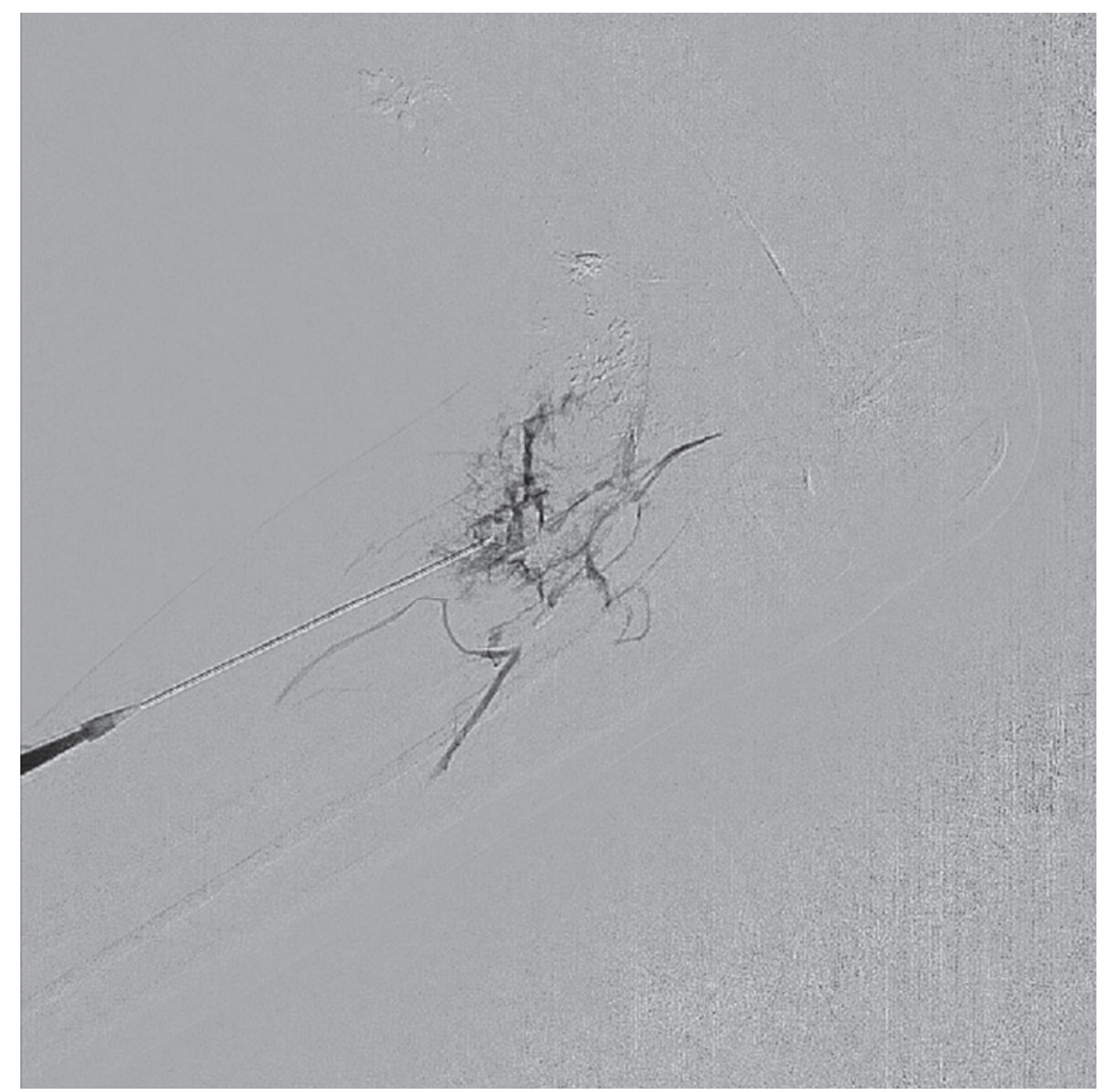

Fig. 3 A 5-year old girl with painful popliteal fossa malformation post multiple previous sclerotherapy treatments. Digital subtraction angiogram image demonstrates fine, irregular channels typical of prior sclerotherapy. Embolization was performed with 1:6 dilution n-BCA:ethiodol.

as the nonionic flush solution. Several hundred milliliters are maintained in a large basin and used to replenish 3-mL D5W flush syringes and a larger (20 mL) D5W "overwash" syringe. Ethiodized oil, $n$-BCA, and potentially contaminated glue "leftovers" are to be maintained in three labeled shot glasses.
A small volume of D5W may be used to rinse the shot glasses of any potential contaminants prior to glue preparation. Ethiodized oil and $n$-NBCA are typically combined in the predetermined ratio, although some interventionalists will wait and use the first venogram for glue ratio determination in the 

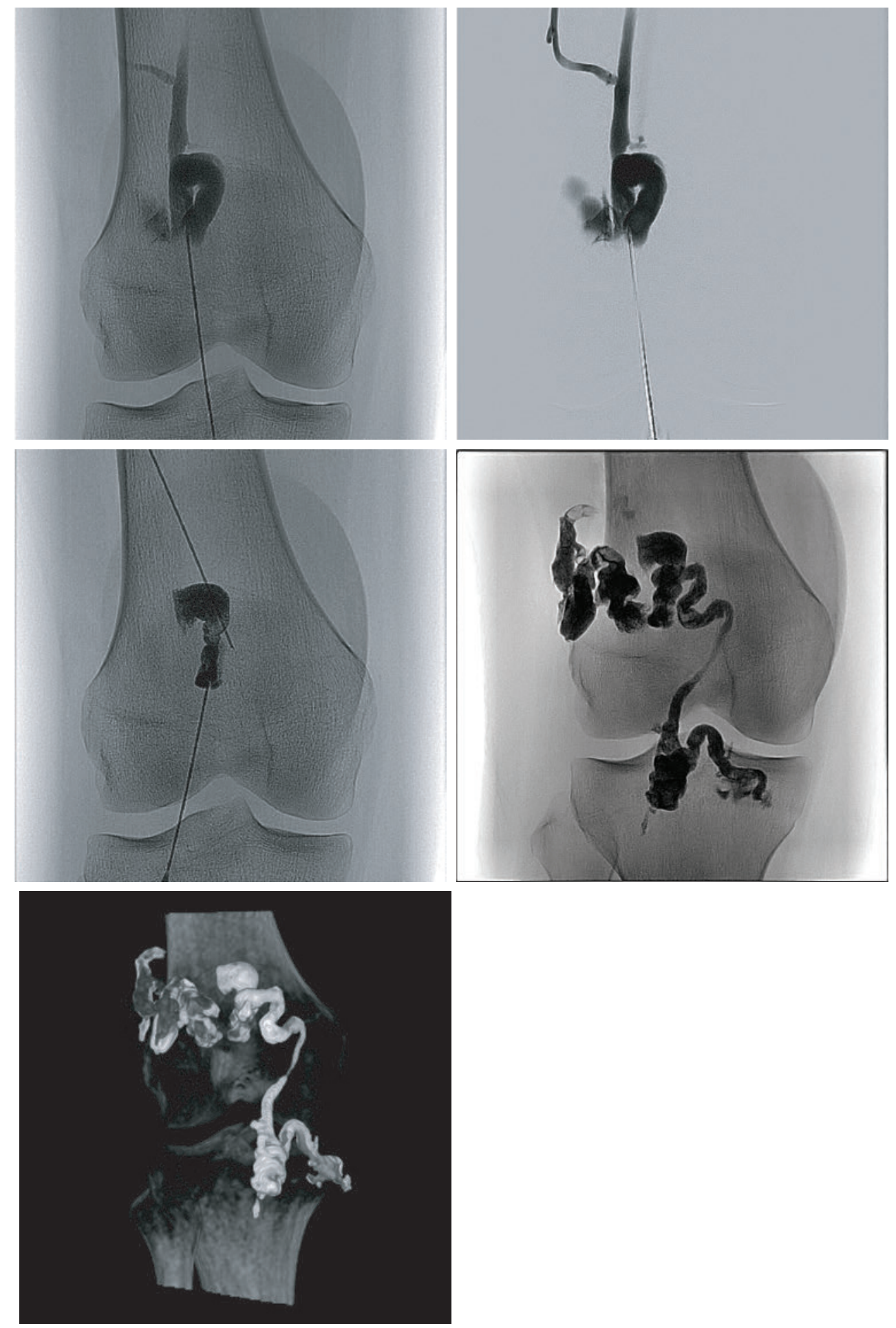

Fig. 4 A 16-year old girl with painful popliteal fossa venous malformation. Initial unsubtracted (a) and subtracted (b) angiogram images demonstrate a broad connection to the popliteal vein. The outflow segment was carefully embolized with 1:2 dilution n-BCA:ethiodol (c) allowing subsequent embolization without egress into the popliteal vein (d). Three-dimensional surface rendering images from a completion cone beam CT (e) were created for surgical guidance.

case of previously treated malformations. Inadequate mixing results in layering of $n$-BCA and ethiodol. Failure to recognize this phenomenon can lead to unexpectedly concentrated or dilute formulations and potential complications. Layering is obvious with blue tinted Histoacryl ( - Fig. 6) but obscure when using colorless Trufill. The selected ratios of $n$-BCA and ethiodol are combined within a shot glass. Thorough stirring with an 18-gauge needle at the time of mixture and immediately before drawing for embolization is recommended regardless of grossly apparent homogeneity. Given the propensity for degradation of plastics by ethiodized oil, the glue mixture should be maintained in glass until immediately before embolization. Polycarbonate syringes are more resistant (but not impervious) to this effect and are readied to serve 


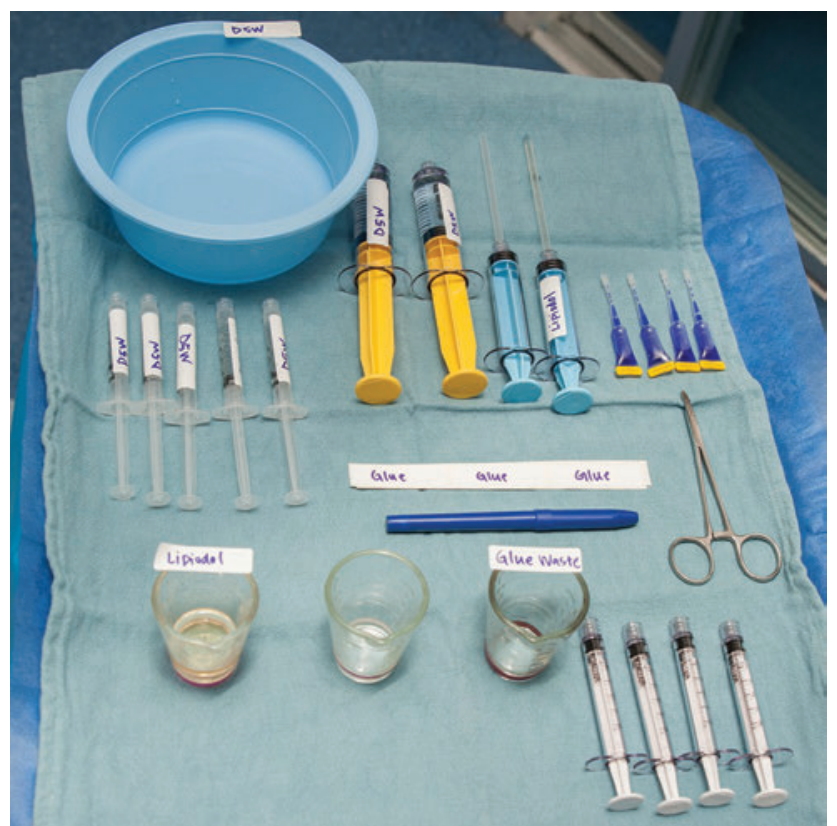

Fig. 5 Glue table. Nonheparinized 5\% dextrose in water (D5W) serves as the nonionic flush solution, maintained in a large basin and used to replenish $3 \mathrm{~mL}$ D5W flush syringes and a larger $(20 \mathrm{~mL})$ D5W "overwash" syringe. Ethiodized oil, $n$-BCA, and potentially contaminated glue "leftovers" are maintained in three labeled shot glasses. Polycarbonate 3 - $\mathrm{mL}$ syringes are used to delivery glue, drawn up after mixing with an 18-gauge needle.

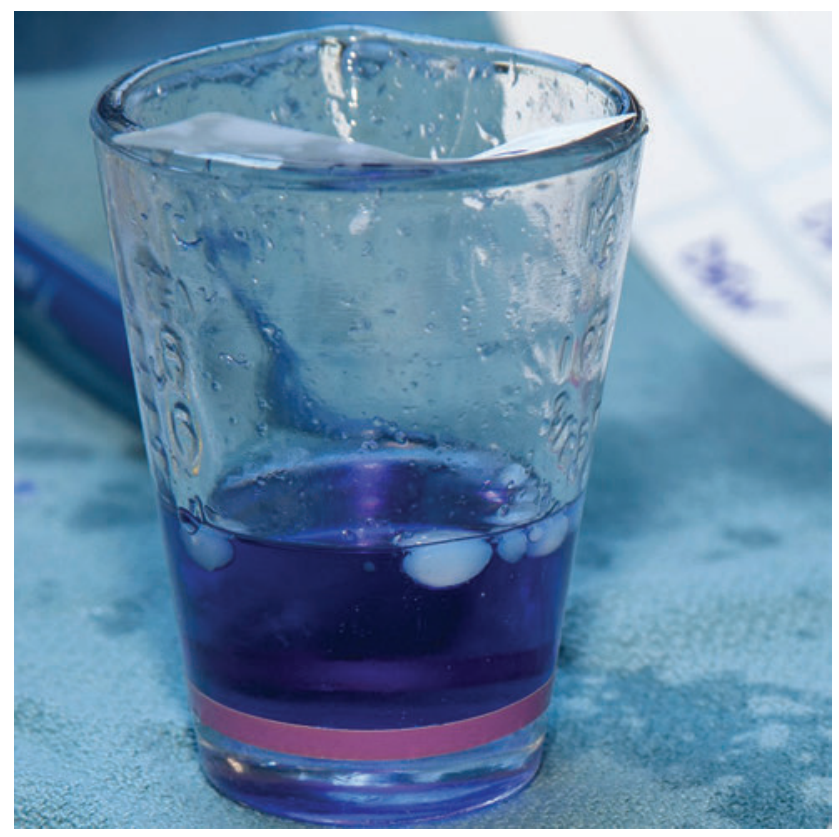

Fig. 7 Contaminated glue mixture. Polymerized glue “icebergs" indicate contamination with ionic solutions.

in embolic delivery with 18 -gauge needles to draw the glue mixture. Floating polymerized glue "icebergs" are the first sign of ionic contamination ( - Fig. 7 ).

A standard procedural table serves the access and angiography aspects of the procedure and is organized in the usual fashion. Winged (butterfly) 23-gauge needles suffice for superficial lesion channel access and have attached tubing. The authors use 21-gauge micropuncture access needles

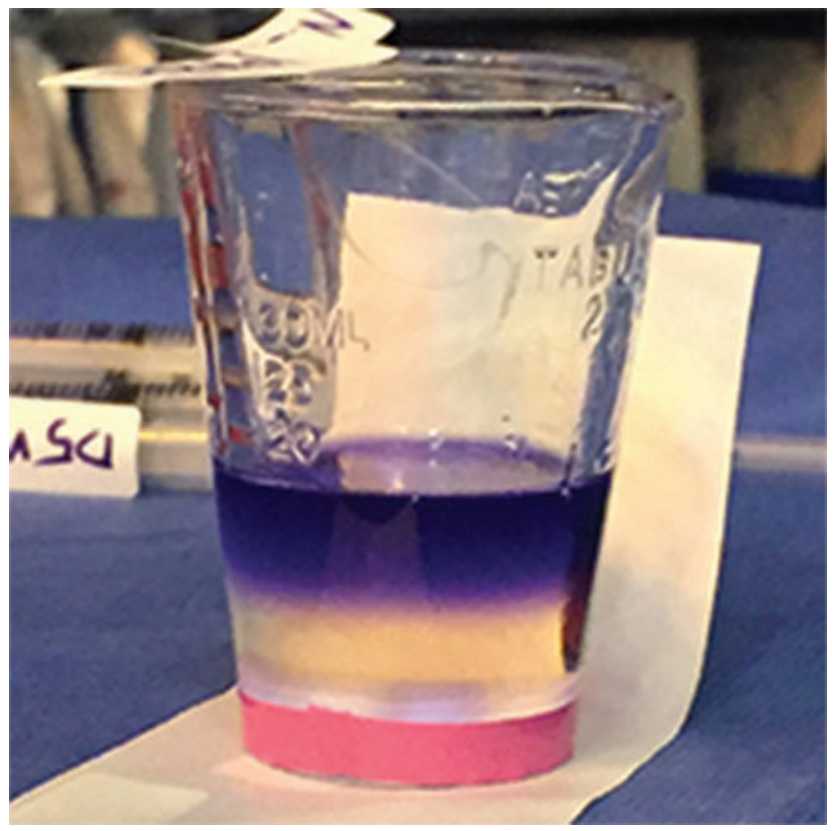

Fig. 6 Layering of ethiodized oil and glue. Inadequately mixed glue made evident by blue-tinted $n$-BCA glue (Histoacryl; B. Braun) layering atop ethiodized oil (Lipiodol; Guerbet USA).

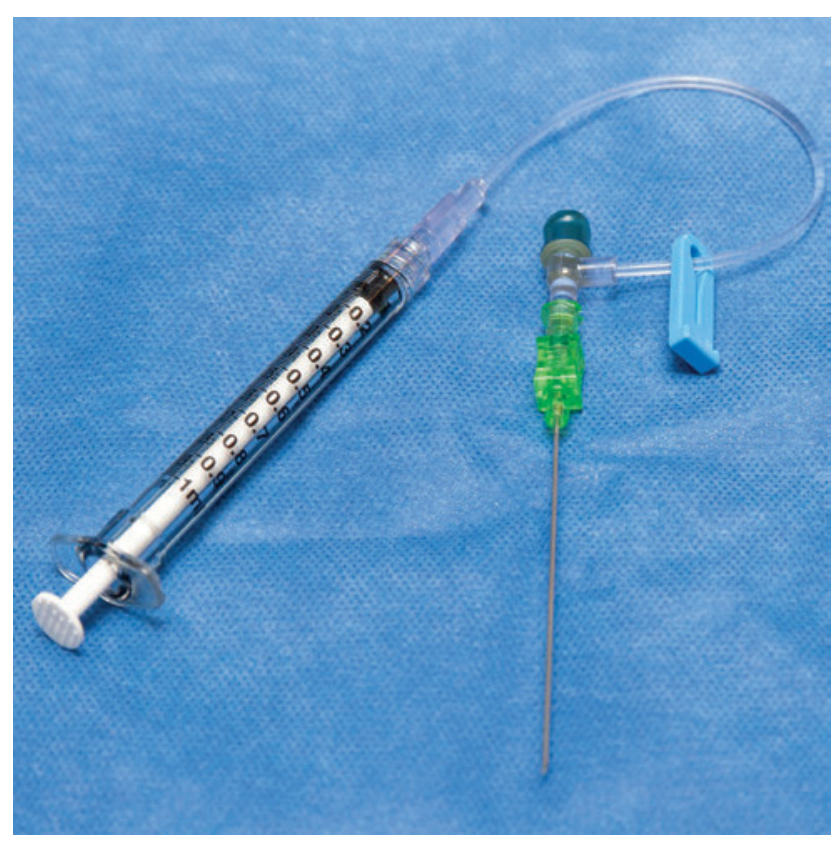

Fig. 8 Access needle and syringe. A 1-mL syringe and tubing attached to a 21-gauge access needle maintains air-free access. The syringe is removed to check for backbleeding after lesion channel access.

for deeper lesion channels, attaching short Luer-Slip tubing. Tubing and needles are preloaded with either saline flush or iodinated contrast media, and then sealed with either tube clamps or 1-mL syringes ( - Fig. 8).

\section{Access and Angiography}

Prior cross-sectional imaging is reviewed. Reference imaging on the fluoroscopy monitor can supplement the lesion shape stored in the mind's eye. Preprocedure ultrasound mapping is performed to map the lesion as well as adjacent macroscopic 
neurovascular structures. Patients are anesthetized and remain sedated through both the embolization and surgical resection. Tourniquets create nonphysiologic conditions and high intralesional pressure, resulting in unpredictable behavior during embolizations; they are not routinely used by the authors.

After sterile preparation and draping, fluoroscopic views are optimized. Saving of table position and image intensifier configuration allows adjustments for more ergonomic ultrasound access but rapid return to positions suited for angiography. Deep or difficult channels are targeted first, anticipating artifactual degradation of the ultrasound images as the embolization proceeds. Selecting a slightly longer-than-necessary access path increases needle stability. The access needle is advanced into the target channel under continuous ultrasound visualization. After sonographic confirmation of lesion entry, the tubing seal is released. Blood return is indicative of malformation (or at least vascular) access. Gentle injection of iodinated contrast media confirms position and grossly highlights both channel capacity and early drainage to conducting veins. Once lesion entry is confirmed, digital subtraction angiography is acquired and then the tubing is again sealed. Images are studied for flow patterns including drainage to normal conducting veins, particularly those leading to the deep venous system. An image best delineating drainage is displayed for reference during the subsequent embolization.

\section{Embolization}

Image subtraction fluoroscopy "negative roadmap" technique is selected with a paired unsubtracted dual fluoroscopy image panel (-Fig. 9). The assistant or technologist readies the D5W flush, D5W overwash, and glue syringes. Glue is once more stirred with the 18-gauge needle before being drawn up into a 3-mL polycarbonate syringe. Drawing only slightly more than the predicted amount needed will conserve glue as the case progresses and minimize procedural costs. Needle access tubing is flushed with a small volume of D5W before connecting the glue syringe. The assistant or technologist uses the overwash D5W syringe to rinse the proceduralist's hands and the catheter hub before and during syringe exchanges, taking care not to make contact and contaminate the syringe or his/her hands. Preparation and communication are essential. Clumsy syringe exchanges allow back bleeding into the access needle tubing, obviating the purpose of the D5W flush and potentiating premature polymerization in the tubing.

Glue is slowly introduced with fluoroscopic imaging commencing as the glue reaches the needle hub. The embolization is as much an art as a science from this stage onward, and a balance must be struck between overzealous (risking extravasation and nontarget embolization) and apprehensive (resulting in premature polymerization and access loss) injections. Maintaining momentum with gentle accelerations and deceleration as indicated is ideal in most circumstances. Constant attention must be paid to known as well as potential previously undocumented draining veins to avoid deep venous thrombosis or central embolization. The accessed lesion channel fills first. With sufficient pressure, glue "bursts" into an adjacent channel of the malformation and the pattern continues, frequently filling large aspects of a malformation from a single access point.

Extension into a draining vein is managed with a slowing or ceasing of the injection but continued observation. Extrinsic compression may be attempted for runaway glue, but vigilant observation should identify nontarget embolization when it is still easily managed with subtle injection pressure adjustments. A 30- to 45-second pause is usually sufficient for polymerization in the draining vein while maintaining a partially liquid state in the malformation. Cautious subsequent increases in the injection pressure often allow ongoing lesion embolization without further expansion into the draining vein.

Periodic resetting of the fluoroscopic subtraction enables subtle detection of changes as the embolization proceeds. A "cap" on embolic within a draining vein after device reset indicates central expansion (-Fig. 10), and embolization should be slowed or stopped. A black boundary or "India ink" appearance after device reset suggests a lesion channel expanding under pressure (-Fig. 11), which may be desirable as the proceduralist cautiously attempts to push glue into adjacent compartments of the lesion. Caution must be exercised as supraphysiologic pressures created may result in surprising egress into previously unseen outflow channels (-Fig. 12). When maximal embolization is achieved, the
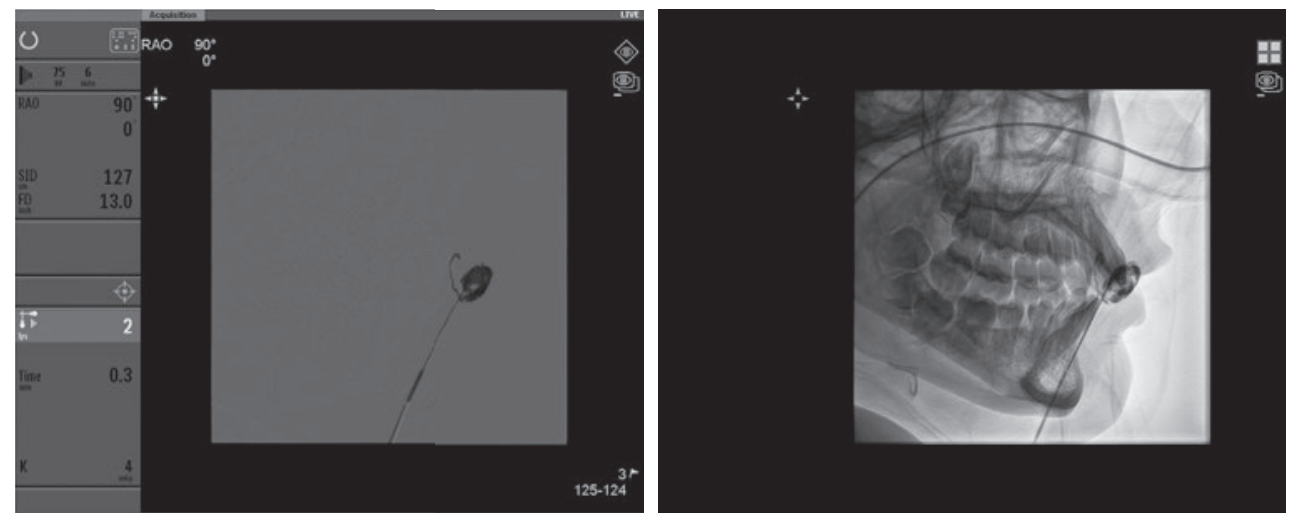

Fig. 9 A 18-year-old woman with a disfiguring venous malformation of upper lip. Screen capture images from the fluoroscopic monitor demonstrate parallel observation of embolic delivery with negative roadmap (a) and reference dual fluoroscopy (b) imaging. 

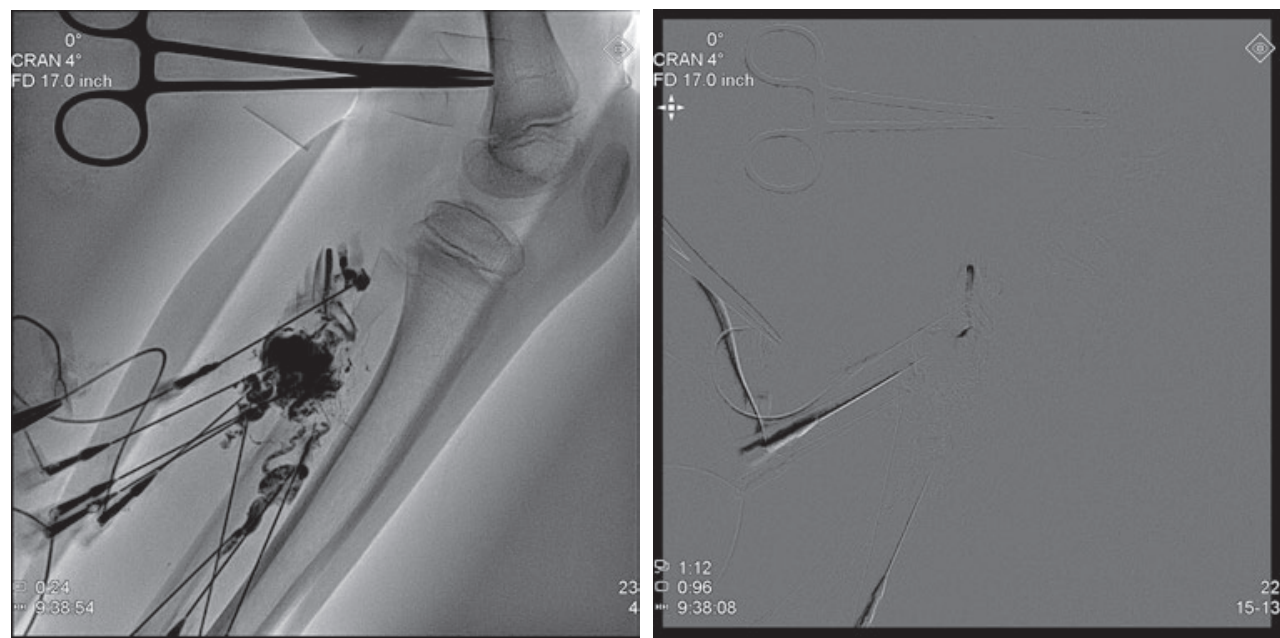

Fig. 10 A 4-year old girl with a painful lower extremity malformation. Negative roadmap imaging following device reset with subtraction of the initial glue installation and propagation into a draining vein (a). A negative roadmap "cap" on the draining vein embolic (b) indicates central expansion, and embolization should be slowed or stopped.
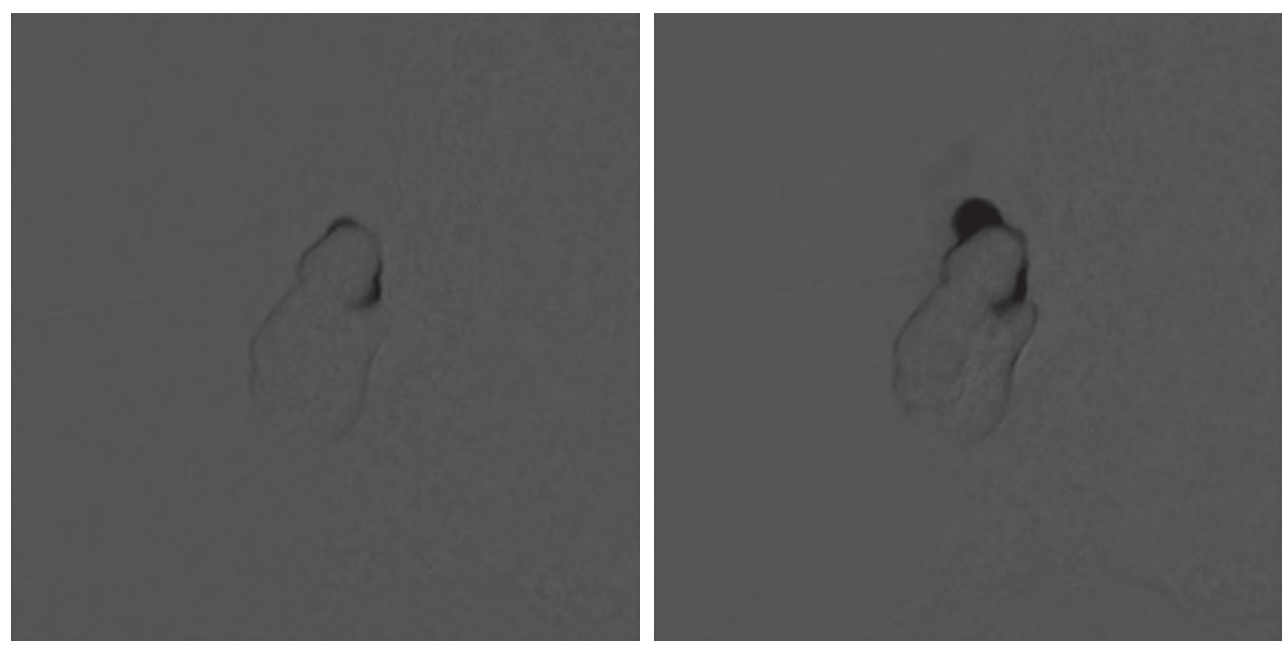

Fig. 11 An 8-year old boy with a painful lower extremity venous malformation. Negative roadmap imaging following device reset with subtraction of the initial glue installation. An "India ink" appearance forms as additional glue causes lesion distension (a) followed by egress into a separate channel as lesional pressure increased $(\mathbf{b})$.
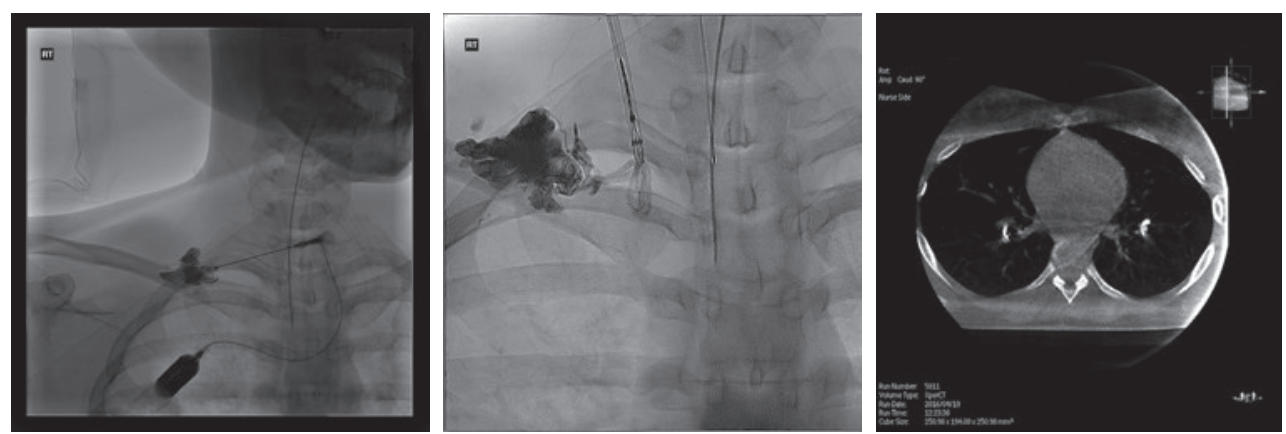

Fig. 12 A 13-year old girl with a painful supraclavicular venous malformation. Preembolization angiogram (a) demonstrated no central drainage and embolization commenced. Increased intralesional pressure resulted in egress of glue from the inferior medial aspects of the malformation into the brachiocephalic vein. Ipsilateral jugular access was successful in snaring and partially removing the nontarget glue (b). However, axial images from a completion cone beam CT (c) demonstrated clinically silent pulmonary embolization of several small fragments.

needle tubing is again clamped. If a significant volume of glue remains in the syringe, this may be potentially salvaged later by directly injecting into the dedicated shot glass for used (potentially contaminated) glue. The glue syringe and D5W flush syringe are discarded to avoid inadvertent return to the "ion-free" glue table.

Attention is then turned to the remaining channels of the malformation that will be accessed, imaged, and embolized in 

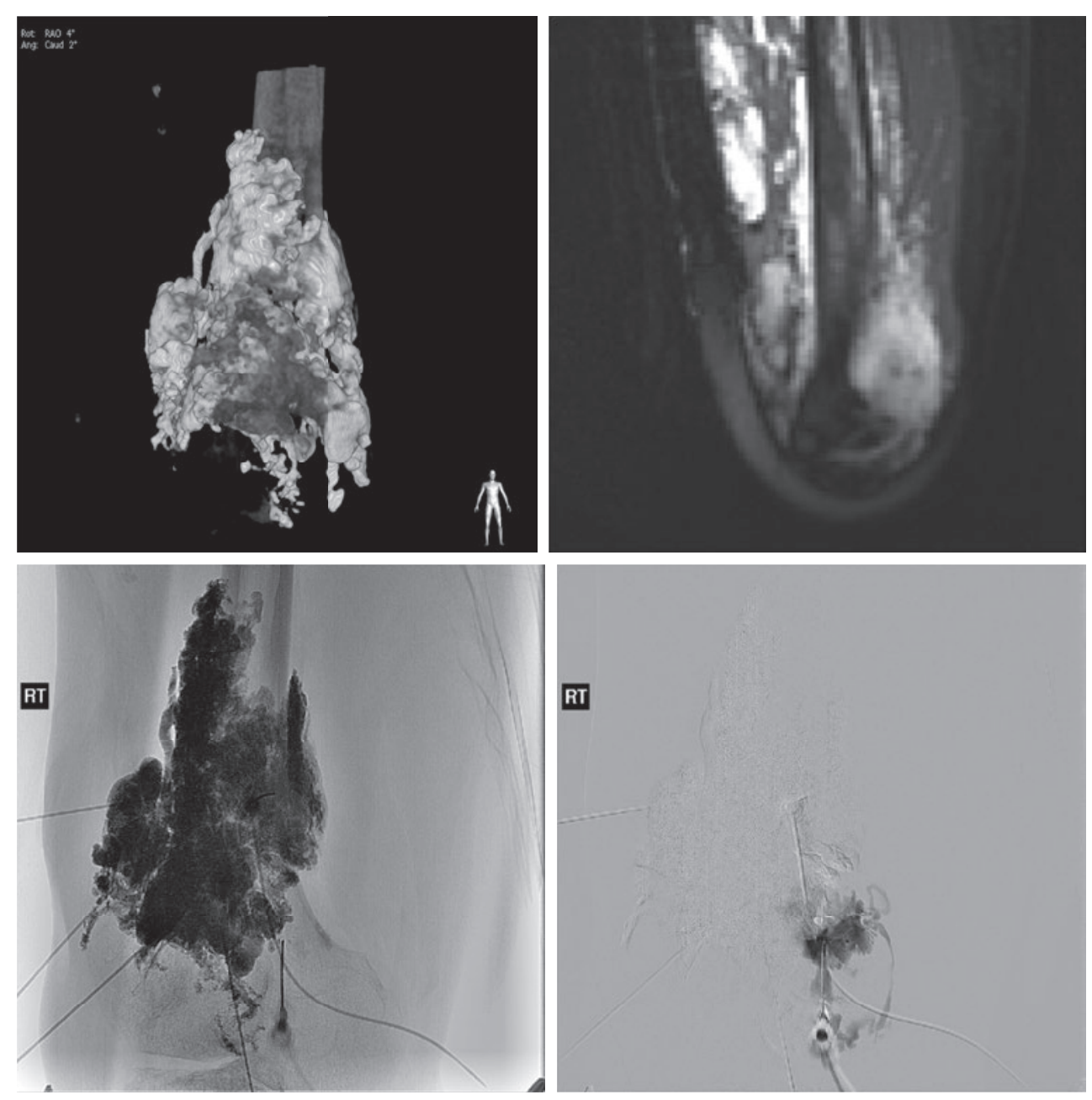

Fig. 13 A 22-year-old woman with a venous malformation of the knee complicated by pain and hemarthrosis. Post embolization three-dimensional surface rendering cone beam CT image (a) demonstrates a photopenic defect at the inferomedial aspect of the malformation. This aspect of the malformation was sonographically obscure but known to be present by STIR sequence images of the preprocedure MR (b). The residual malformation was targeted fluoroscopically, imaged (c), and embolized (d) to complete the procedure.

the same fashion. In the minutes after one region of treatment is performed, tissue manipulations such as ultrasound scanning should be performed gently to avoid massaging still-liquid glue unpredictably out of the embolized channels. Needles should remain in place for a minimum of 10 minutes to minimize glue extravasation after needle removal. Occasionally, the easily accessible channels of a malformation will contain thrombus or phlebolith. Though this limits backbleeding confirmation of lesion entry, access followed by gentle hydrodissection or thrombus fracture will enable imaging and embolization. Rarely, residual patent channels will be sonographically obscure but obvious by a photopenic defect in the fluoroscopic appearance of the lesion. If the region is known to be void of critical structures, this aspect can be targeted with computed tomography (CT) or fluoroscop-

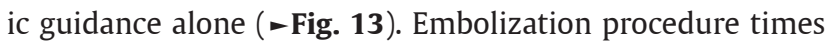
vary widely from 30 minutes for previously untreated focal, cavitary subtypes to 2 hours for infiltrating lesions with complex architecture or lesions with small isolated channels as a result of prior sclerotherapy.

\section{Completion Imaging}

Fluoroscopic imaging after each embolization stage tracks progression until the glue cast grossly resembles the entirety of the lesion targeted for excision. Although channel distension commonly results in minor geometric distortion, an excellent spatial match to previous imaging should be sought. After a complete fluoroscopic appearance is achieved, the authors acquire a cone beam CT of the region of interest. Multiplanar reformations compared side by side to fluid sensitive sequences of previous magnetic resonance studies provide confident confirmation of total embolization. Three-dimensional surface renderings, particularly for lesions interdigitating between unresectable anatomy, made available before surgical excision are helpful to the surgical colleagues, particularly intraoperatively ( - Fig. 4 ). 
At embolization completion, the patient is transferred under anesthesia to the operating room.

\section{Conclusion}

Venous malformations may be treated with surgical excision or sclerotherapy. Though these options can provide symptomatic relief, multiple treatment rounds are often required for full therapeutic benefit. Each procedure brings additional costs, risks, and a protracted treatment schedule. Preoperative glue embolization of VMs followed immediately by surgical excision provides definitive treatment in a single session.

This single-stage procedure requires extensive communication and coordination between multiple teams, namely interventional radiology, surgery, and anesthesia, which may not be feasible at every institution. Because of diffuse disease or comorbidities, some patients will not be good candidates for preoperative glue embolization. Still others may decide they would prefer multiple rounds of sclerotherapy rather than undergoing surgery and having a scar. Nonetheless, this procedure offers an appealing alternative to sclerotherapy or surgery alone. In experienced hands, it is safe and has a high technical success rate. It limits rounds of treatment and therefore radiation exposure. Additionally, this technique dramatically reduces intraoperative blood loss and makes excision less technically challenging by defining lesion margins.

\section{Conflict of Interest}

The authors have no financial disclosures or conflicts of interest.

\section{References}

1 Burrows PE, Mason KP. Percutaneous treatment of low flow vascular malformations. J Vasc Interv Radiol 2004; 15(5):431-445
2 Burrows PE. Management of low-flow vascular malformations. In: Mauro MA, Murphy KPJ, Thomson KR, Venbrux AC, Morgan RA, eds. Image-Guided Interventions. Philadelphia, PA: Elsevier Saunders; 2014:284-290

3 Cahill AM, Nijs ELF. Pediatric vascular malformations: pathophysiology, diagnosis, and the role of interventional radiology. Cardiovasc Intervent Radiol 2011;34(4):691-704

4 Qiu Y, Chen H, Lin X, Hu X, Jin Y, Ma G. Outcomes and complications of sclerotherapy for venous malformations. Vasc Endovascular Surg 2013;47(6):454-461

5 Rabe E, Pannier F. Sclerotherapy in venous malformation. Phlebology 2013;28(Suppl 1):188-191

6 Roh YN, Do YS, Park KB, et al. The results of surgical treatment for patients with venous malformations. Ann Vasc Surg 2012;26(5):665-673

7 Bowman J, Johnson J, McKusick M, Gloviczki P, Driscoll D. Outcomes of sclerotherapy and embolization for arteriovenous and venous malformations. Semin Vasc Surg 2013;26(1):48-54

8 van der Vleuten CJM, Kater A, Wijnen MHWA, Schultze Kool LJ, Rovers MM. Effectiveness of sclerotherapy, surgery, and laser therapy in patients with venous malformations: a systematic review. Cardiovasc Intervent Radiol 2014;37(4):977-989

9 Tieu DD, Ghodke BV, Vo NJ, Perkins JA. Single-stage excision of localized head and neck venous malformations using preoperative glue embolization. Otolaryngol Head Neck Surg 2013;148(4):678-684

10 Chewning R, Lindberg A, Monroe E, Vaidya S, Shivaram G. Glue embolization of pediatric non-head and neck venous malformations. J Vasc Interv Radiol 2016;3(27):S190

11 Rosen RJ, Contractor S. The use of cyanoacrylate adhesives in the management of congenital vascular malformations. Semin Intervent Radiol 2004;21(1):59-66

12 PollakJS, White RIJr. The use of cyanoacrylate adhesives in peripheral embolization. J Vasc Interv Radiol 2001;12(8):907-913 